\title{
Near-complete regression 19 years after Gamma Knife radiosurgery of vestibular schwannoma with massive pseudoprogression: case report
}

\author{
Lai-fung Li, MRes(Med), FRCSEd(SN), ${ }^{1}$ Chung-ping Yu, FRCS, FHKAM, ${ }^{2}$ \\ Anderson Chun-on Tsang, MBBS, FRCSEd(SN), ${ }^{1}$ Benedict Beng-teck Taw, MMedSc, FRCSEd(SN), ${ }^{1}$ \\ and Wai-man Lui, FRCS, FHKAM ${ }^{1}$
}

'Division of Neurosurgery, Department of Surgery, Li Ka Shing Faculty of Medicine, The University of Hong Kong; and ${ }^{2}$ The Brain Center, Canossa Hospital, Hong Kong

\begin{abstract}
Gamma Knife radiosurgery (GKRS) is a frequent treatment choice for patients with small- to moderate-sized vestibular schwannoma (VS). However, pseudoprogression after GKRS is commonly observed, with a reported incidence ranging from $7 \%$ to $77 \%$. The wide range of the reported incidence of pseudoprogression reflects the fact that there is no consensus on how it should be diagnosed.

The authors present the case of a 66-year-old woman who had a 2.5 -cm right-sided VS treated with GKRS in 1997. The first posttreatment MRI obtained 5 months later showed that the tumor volume had increased to $9.7 \mathrm{~cm}^{3}$. The tumor volume increased further and reached its peak 24 months after treatment at $20.9 \mathrm{~cm}^{3}$, which was a $161 \%$ increase from pretreatment volume. Thereafter, the tumor shrank gradually and mass effect on the brainstem reduced over time. By 229 months after treatment, the tumor volume was $1.0 \mathrm{~cm}^{3}$, equaling $12.5 \%$ of pretreatment tumor volume, or $4.8 \%$ of peak tumor volume after treatment. This case demonstrates that if a patient remains asymptomatic despite a dramatic increase in tumor volume after GKRS, observation remains an option, because even a very sizable tumor can shrink with near-complete resolution. Patients undergoing GKRS for VS should be counseled regarding the possibility of pseudoprogression, and followed carefully over time while avoiding premature decisions for surgical removal after treatment. https://thejns.org/doi/abs/10.3171/2020.3.JNS20389
\end{abstract}

KEYWORDS vestibular schwannoma; Gamma Knife; pseudoprogression; stereotactic radiosurgery

$\mathrm{G}$ AMMA Knife radiosurgery (GKRS) is a frequent treatment choice for patients with small- to moderate-sized vestibular schwannoma (VS) because of its noninvasiveness and better preservation of facial nerve function and hearing. ${ }^{1}$ However, transient tumor growth after treatment (i.e., pseudoprogression) is commonly observed, with a reported incidence ranging from $7 \%$ to $77 \% .^{2}$ The wide range of reported incidence reflects the fact that there is no consensus on diagnosing pseudoprogression or, conversely, identifying patients who experience treatment failure after GKRS for VS. Nevertheless, in the face of lesion enlargement on posttreatment MRI, clinicians have to decide whether to continue observation or proceed to surgery. These decisions are by and large based on personal experience, development of new symptoms, size of the enlarging tumor, and patient concerns regarding these imaging changes.

The published literature on pseudoprogression reports the incidence, time to onset, time to peak volume, maximum volume change, and the percentage of volume change per year as aids for diagnosis and subsequent decision-making. ${ }^{2-6}$ But these reports are mainly of small tumors, so that even after a significant percentage volume increase, their size was unlikely to cause new symp- 

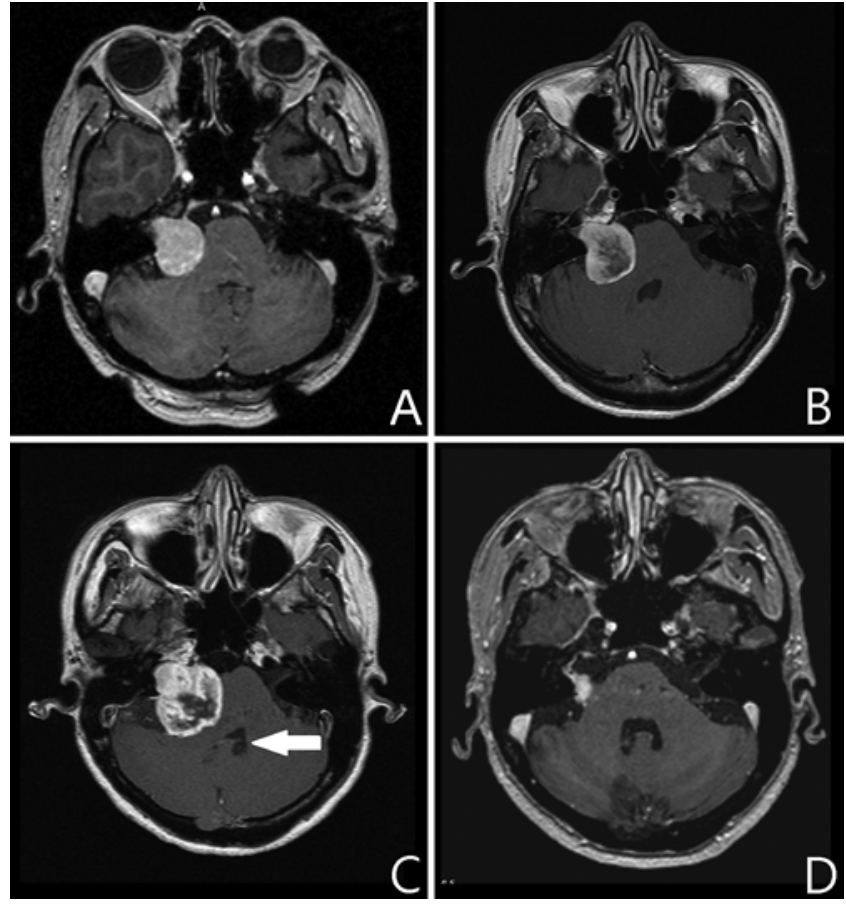

FIG. 1. Serial Gd contrast-enhanced T1-weighted axial MRI sequences showing the tumor at different times. A: December 1997-pretreatment, $8.0 \mathrm{~cm}^{3}$. B: May $1998-5$ months after treatment, $9.7 \mathrm{~cm}^{3}$. C: May 1999-24 months after treatment, $20.9 \mathrm{~cm}^{3}$. White arrow, distorted fourth ventricle. D: January $2017-225$ months after treatment, $1.0 \mathrm{~cm}^{3}$. toms. On the other hand, it has been shown that the timing of tumor regression is highly variable, and that $32 \%$ of tumors regress between the 4th and 6th years after treatment. ${ }^{2}$ Therefore, a decision based only on the percentage of volume increase but not on the actual tumor volume that patients can tolerate may lead to an early diagnosis of treatment failure, and result in unnecessary surgery in a patient with what is actually a tumor that is showing only rapid pseudoprogression. Herein we report on a patient with an initially moderate-sized VS with dramatic pseudoprogression after GKRS, who did not have surgery and went on to have near-complete tumor regression that has remained stable 19 years after treatment.

\section{Case Report}

Our patient is now 66 years old. Her history dates back to 1997, when she presented with decreased hearing in her right ear but preserved speech discrimination. MRI with Gd contrast-enhanced T1-weighted sequence showed a 2.5-cm-diameter, right cerebellopontine angle, contrastenhancing tumor with extension to the internal acoustic meatus and abutting the brainstem. The radiological diagnosis was a right-sided VS. After discussion of treatment options the patient decided to receive GKRS. She was treated uneventfully on December 2, 1997, using a Model B Gamma Knife unit (Elekta Instruments AB). The tumor volume was $8.0 \mathrm{~cm}^{3}$ and the margin dose was $10 \mathrm{~Gy}$ to the $40 \%$ isodose line (Figs. 1A and 2). The first posttreatment MRI sequence obtained 5 months later showed decreased
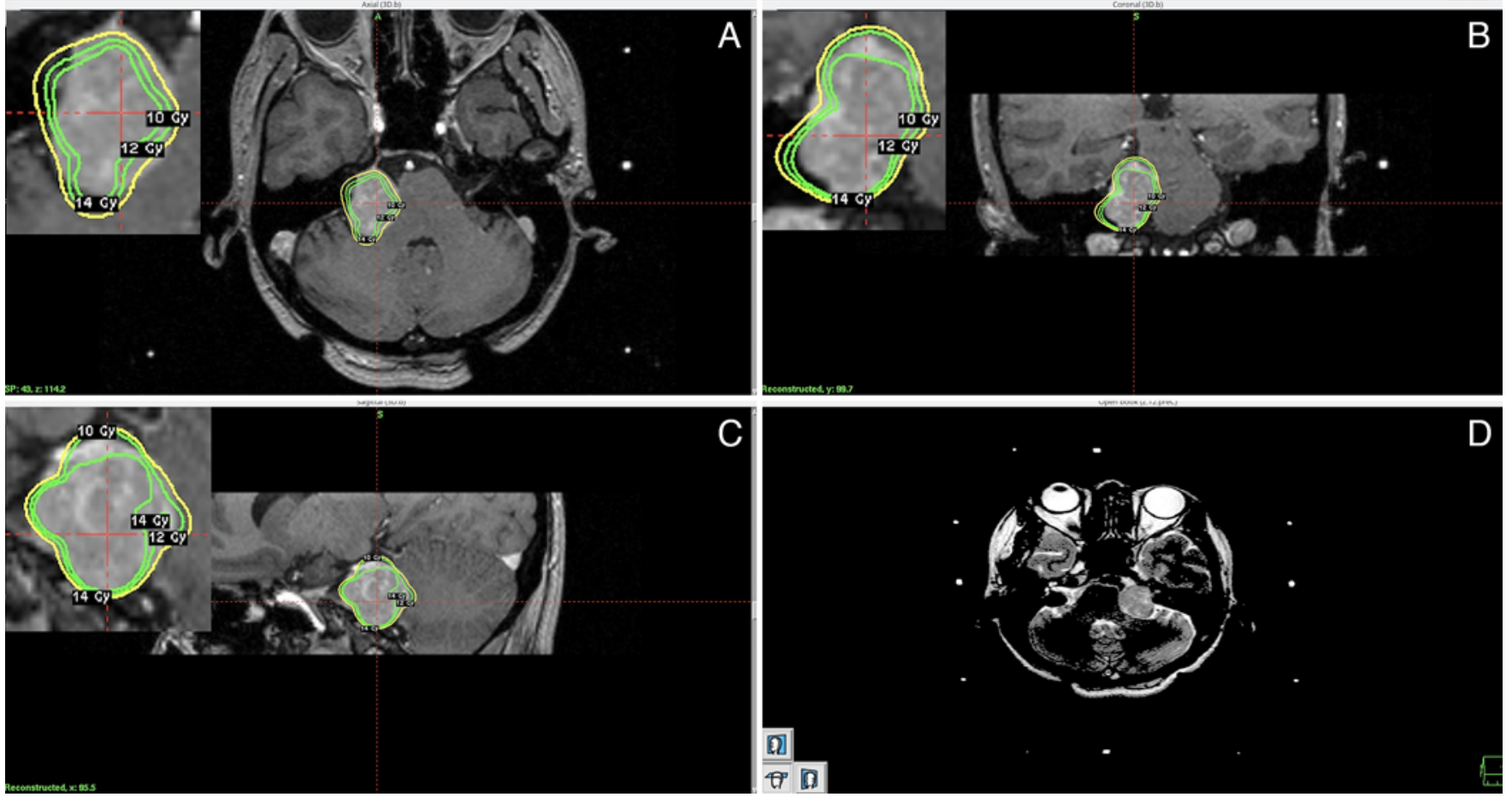

FIG. 2. Treatment planning images. Prescription dose: $10 \mathrm{~Gy}$ at $40 \%$ isodose line. Treatment volume $8.038 \mathrm{~cm}^{3} ; 10 \mathrm{~Gy}, 12 \mathrm{~Gy}$, and 14 Gy covering $91.9 \%, 75 \%$, and $55.3 \%$ of tumor, respectively. Figure is available in color online only. 


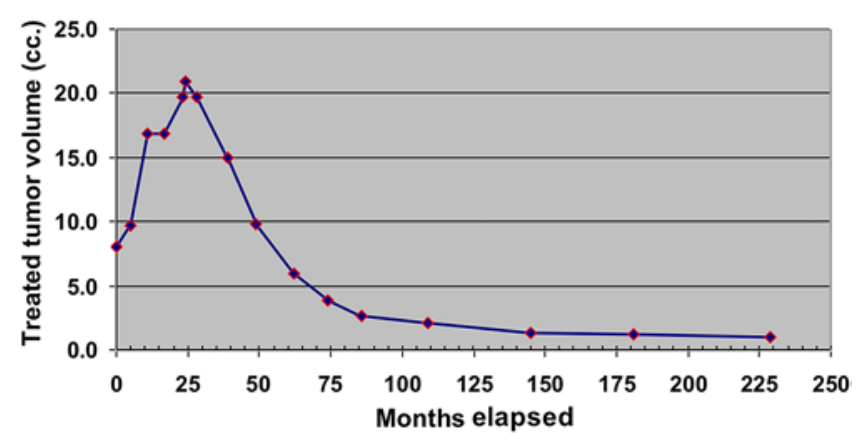

FIG. 3. Graph showing treated tumor volume $\left(\mathrm{cm}^{3}\right)$ versus months elapsed after GKRS. Figure is available in color online only.

contrast enhancement and tumor volume increased to $9.7 \mathrm{~cm}^{3}$ (Fig. 1B). After that, the patient underwent reassessment MRI annually. The tumor volume increased further and reached its peak 24 months after treatment at $20.9 \mathrm{~cm}^{3}$, which was a $161 \%$ increase from the pretreatment volume (Fig. 1C). The tumor also caused increased mass effect on the brainstem with distortion of the fourth ventricle. Because the patient had no new symptoms as a result of tumor enlargement, she was observed and surgical treatment was withheld. Thereafter, the tumor shrank gradually and mass effect on the brainstem likewise reduced. By January 2017 (i.e., 229 months after treatment), the tumor volume was $1.0 \mathrm{~cm}^{3}$, which was $12.5 \%$ of the pretreatment tumor volume, or $4.8 \%$ of the peak tumor volume after GKRS (Figs. 1D and 3). The tumor was no longer abutting the brainstem, and patient has remained clinically stable throughout these years.

\section{Discussion}

Our patient showed features compatible with the reported characteristics of VS pseudoprogression after GKRS. The tumor lost its contrast enhancement soon after treatment, with rapid enlargement in the first 2 years. Multiple reviews have shown that tumors with pseudoprogression experience an early and more rapid growth compared to tumors in treatment failure groups. ${ }^{2-6}$ It has been reported that $90 \%$ of tumors had peaked in size by 3.5 years following treatment. ${ }^{2}$ However, early and rapid tumor enlargement can still be occasionally observed in treatment failure groups..$^{2,4,5}$ As a result, Hayhurst and Zadeh suggested that only late enlargement beyond 24 months is indicative of failure, and that no decisions for possible retreatment should be made for at least 36 months. ${ }^{4}$ However, late enlargement together with late regression has also been observed in a pseudoprogression group. ${ }^{2}$ Therefore, these reported characteristics are not completely reliable for decision-making. The key point is that clinicians should allow sufficient time once lesion enlargement is seen after GKRS to allow for pseudoprogression to become manifest by shrinkage of the lesion.

It has been reported that tumors with more than a $300 \%$ volume increase after GKRS can diminish afterward. ${ }^{2}$ However, the pretreatment volume of the patient's tumor was not reported in the study by Breshears et al. Our pa- tient did not have the largest reported percentage of volume change, but her tumor's pretreatment volume almost reached the limit of GKRS feasibility. With a peak posttreatment volume of $20.9 \mathrm{~cm}^{3}$, mass effect on the brainstem, and fourth ventricle distortion, it was tempting to consider this a treatment failure and to proceed with surgery to prevent new neurological deficits. Resection after radiosurgery has been described to be more or less difficult, or similar to nonirradiated tumors. ${ }^{7}$ The main issue is facial nerve preservation, which often can be maintained when resection is performed by experienced surgeons. Our case demonstrates that if a patient remains asymptomatic despite alarming radiological findings, observation remains an option. Provided there is a long enough observation time, a very sizable tumor can shrink with near-complete resolution. This case also demonstrates that the percentage of maximal volume change and actual tumor peak volume are not indicative of treatment failure. Only sufficient time can help to distinguish between pseudoprogression and genuine progression.

Most importantly, patients should be well informed about the possibility of pseudoprogression and related imaging changes when being counseled regarding GKRS. The psychological pressure on a patient of having a rapidly enlarging tumor after treatment can only be assuaged by a good doctor-patient relationship and the patient's trust in their doctor's judgment. This also emphasizes the need for long-term follow-up by the treating physician who knows the patient best.

\section{Conclusions}

Dramatic enlargement of a VS after GKRS, even to the point of fourth ventricular distortion, can still reflect pseudoprogression. In the absence of new symptoms, patients with this finding can be observed with serial MRI and clinical examination, and not undergo unnecessary and potentially morbidity-producing surgery.

\section{References}

1. Pollock BE. Vestibular schwannoma management: an evidence-based comparison of stereotactic radiosurgery and microsurgical resection. Prog Neurol Surg. 2008;21:222-227.

2. Breshears JD, Chang J, Molinaro AM, et al. Temporal dynamics of pseudoprogression after Gamma Knife radiosurgery for vestibular schwannomas - a retrospective volumetric study. Neurosurgery. 2019;84(1):123-131.

3. Delsanti C, Roche PH, Thomassin JM, Régis J. Morphological changes of vestibular schwannomas after radiosurgical treatment: pitfalls and diagnosis of failure. Prog Neurol Surg. 2008;21:93-97.

4. Hayhurst C, Zadeh G. Tumor pseudoprogression following radiosurgery for vestibular schwannoma. Neuro Oncol. 2012;14(1):87-92.

5. Pollock BE. Management of vestibular schwannomas that enlarge after stereotactic radiosurgery: treatment recommendations based on a 15 year experience. Neurosurgery. 2006;58(2):241-248.

6. Yu CP, Cheung JY, Leung S, Ho R. Sequential volume mapping for confirmation of negative growth in vestibular schwannomas treated by gamma knife radiosurgery. $\mathrm{J} \mathrm{Neu-}$ rosurg. 2000;93(suppl 3):82-89.

7. Pollock BE, Lunsford LD, Kondziolka D, et al. Vestibu- 
lar schwannoma management. Part II. Failed radiosurgery and the role of delayed microsurgery. J Neurosurg. 1998;89(6):949-955.

\section{Disclosures}

The authors report no conflict of interest concerning the materials or methods used in this study or the findings specified in this paper.

\section{Author Contributions}

Conception and design: Li, Yu. Acquisition of data: Yu. Analysis and interpretation of data: Yu. Drafting the article: Li. Critically revising the article: $\mathrm{Li}, \mathrm{Yu}$, Tsang. Reviewed submitted version of manuscript: $\mathrm{Li}, \mathrm{Yu}$, Tsang, Taw. Approved the final version of the manuscript on behalf of all authors: Li. Administrative/technical/ material support: Li, Yu. Study supervision: Yu, Lui.

\section{Correspondence}

Lai-fung Li: The University of Hong Kong, Hong Kong. llfrandom@gmail.com. 